\title{
FLOW SINGULARITY AND SLIP VELOCITY IN PLANE EXTRUDATE SWELL COMPUTATIONS
}

\author{
R.D. WESSON and TASOS C. PAPANASTASIOU \\ Department of Chemical Engineering, The University of Michigan, Ann Arbor, \\ Michigan 48109 (U.S.A.)
}

(Received November 3, 1986; in revised form July 29, 1987)

\section{Summary}

It is common knowledge that flows of viscoelastic liquids with stress singularities, like the extrudate swell flow, pose formidable obstacles to numerical computations at relatively low Weissenberg number. This paper describes an effort toward alleviating the stress singularity by means of a slip boundary condition at the die wall. The Oldoyd-B and the upper-convected Maxwell differential constitutive equations were used for simplicity and computational efficiency. With a no-slip boundary condition it was found that for Newtonian, upper-convected Maxwell and Oldroyd-B liquids the global solution was always mesh-dependent until the Newton iteration diverged at very fine tessellations in the vicinity of the static contact line. With a natural slip boundary condition the global solution became mesh-independent at the same tessellations. Moreover, the macroscopic predictions became independent of the amount of slip in a relatively broad region of slip coefficient. The Newton iteration converged up to Weissenberg number 0.6 with a no-slip boundary condition and up to 1.7 with a slip boundary condition for the upper-convected Maxwell liquid. For the Oldroyd-B liquid the maximum Weissenberg number was 0.85 without slip and 1.866 with slip. Although slip velocity, surface tension and Newtonian viscosity (or retardation time) enhanced some numerical stability in general, it appears unlikely that they could advance viscoelastic computations significantly. In the limiting case of no swelling, at infinitely large surface tension, the analytical solution for Newtonian and, a second order fluid showed:

(a) elasticity increases the strength of the singularity that exists for Newtonian liquid at the contact line, and thus Newton iteration is expected to diverge at coarser and coarser tessellations as the elasticity increases in agreement with the finite element findings. 
(b) Finite element predictions for the same flow agreed with the analytical solution in the vicinity of the singularity only when a slip boundary condition was employed.

(c) Slip boundary condition in the vicinity of the contact line alleviates the stress singularity. However, it forces the stress to go through a maximum which is equally catastrophic of the Newton iteration convergence.

\section{Introduction}

In flowing Newtonian or viscoelastic liquids the stress grows large near geometrical singularities (e.g. contraction flow) near static contact lines (e.g. extrudate swell flow) and near dynamic contact lines (e.g. curtain, slide and extrusion coating). In fact, the stress at these vicinities is made artificially infinitely large because the mathematical representation of the boundary condition fails to account for the microscale physics of the wetting there. The singular stress causes numerical computations to diverge when the tessellation is made finer and finer in order to achieve a mesh independent solution. In viscoelastic liquids the stress grows even larger because of the extensional character of the flow in the vicinity of a singularity.

The divergence of the Newton (or any other) iteration is common in calculations with both differential [1-3] and integral [4-6] constitutive equations. The behavior of Newton iteration at about the divergence gave rise to speculations of turning points in the parametric space of Weissenberg number. Yeh et al. [7], investigated and proved the existence of these points in the contraction flow. Keunings [8] concluded that a limit point exists which goes to zero Weissenberg after extensive mesh refinement in a contraction flow. Papanastasiou et al. [6] concluded that these points were artificially induced by the inability of the constitutive equation to account for the discontinuous deformation about the singularity in the extrudate swell flow and thus abandoned the idea of pursuing them. The solutions in Refs. 1-8 were all obtained with a no-slip boundary condition at the die-wall.

Silliman and Scriven [9] investigated the extrudate swell flow of a Newtonian liquid with and without slip. They found that unlike the no-slip boundary condition a natural slip boundary condition (relation between shear stress and velocity at wall) led to bounded stresses and thus to solution independence of the tessellation. Moreover, for a wide range of slip coefficient values (constant of proportionality between stress and velocity at wall) the flow was affected only locally and the macroscopic predictions of extrudate swelling remained uninfluenced. Following them, Papanastasiou et 
al. [6] introduced slip in their numerical computations with an integral constitutive equation and observed that slip, as expressed by a linear relation between the shear stress and the velocity in the vicinity of the singularity, led to calculations at Weissenberg numbers higher than those obtained under the same circumstances with a no-slip boundary condition. At that point the computational cost with integral constitutive equation was formidable and no extensive studies on slip and its significance to viscoelastic calculations were made.

The slip of polymer melts in capillaries is experimentally justified [10]. Very recently Cohen and Metzner [11] reported results from experiments with several polymer solutions in capillaries and concluded with an empirical relation

$V_{\mathrm{a}}=\alpha \tau_{\mathrm{w}}^{m}$

to model apparent slip of polymeric solutions. Here $V_{\mathrm{s}}$ and $\tau_{\mathrm{w}}$ are the slip velocity and the wall shear stress respectively. The exponent $m$ and the coefficient $\alpha$ depended on the elasticity of the liquid and the magnitude of the shear stress $\tau_{w}$ at the wall. Equation (1) can be used as a working hypothesis for slip in general independently of obvious differences in the physics between the behavior of solutions and melts.

In this work we investigate the significance of slip, as expressed by eqn. (1), on the accuracy of the predictions and on the numerical stability of extrudate swell flow calculations for Newtonian and viscoelastic liquids. The main goal is to alleviate the singular stress by means of eqn. (1) and achieve solutions at Weissenberg numbers higher than those obtained without slip. The effects of retardation time or Newtonian viscosity and surface tension are also investigated. The numerical prediction are supplemented by analytical results in the case of a stick-slip flow of a second order fluid.

\section{Governing equations}

The geometry of the flow is shown in Fig. 1. A sheet of liquid issuing from a slit at zero Reynolds number swells owing to the abrupt change in boundary condition at the exit of the slit. The governing conservation equations are

$\nabla \cdot \boldsymbol{u}=\mathbf{0}$

$\nabla \cdot(-p I+\tau)=0$.

The system closes by the constitutive equation of Oldroyd-B liquid

$\tau+\lambda_{1} \dot{\tau}=2 \mu\left(D+\lambda_{2} \dot{D}\right)$ 
or equivalently [2] with

$\tau=\tau_{1}+\tau_{2}$,

$\tau_{1}+\lambda_{1} \dot{\tau}_{1}=2 \mu_{1} D$,

$\tau_{2}=2 \mu_{2} D$,

$\lambda_{2}=\lambda_{1} \frac{\mu_{2}}{\mu_{1}+\mu_{2}}, \mu=\mu_{1}+\mu_{2}$.

Here $\lambda_{1}$ and $\lambda_{2}$ are the relaxation and retardation times respectively, and $\mu$ the zero shear viscosity. Commonly $\mu_{1}=8 \mu / 9, \mu_{2}=\mu / 9$ and thus $\lambda_{2}=\lambda_{1} / 9$. The superscript dot denotes upper convected derivatives of stress or rate of strain [2]. The integral analogue of eqns. (5a) to (5c) is

$\tau=2 \mu_{2} D+\int_{-\infty}^{t} \frac{\mu_{1}}{\lambda_{1}^{2}} \exp \left(-\frac{t-t^{\prime}}{\lambda_{1}}\right)\left(B_{t}\left(t^{\prime}\right)-I\right) \mathrm{d} t^{\prime}$

where $\boldsymbol{B}_{t}\left(t^{\prime}\right)$ is the Finger tensor of past states $t^{\prime}$ relative to the present state at $t$, and so eqn. (4) models solutions of viscoelastic liquid of relaxation time $\lambda_{1}$ and constant viscosity $\mu_{1}$ in a Newtonian solvent of viscosity $\mu_{2}$. Thus if eqn. (6) is to be adopted eqn. (1) is appropriate too.

To calculate the location of the free surface the kinematic condition

$\boldsymbol{n} \cdot \boldsymbol{u}=\mathbf{0}$

that forbids mass penetration across the free surface of normal unit vector $n$ is solved simultaneously with eqns. (2), (3), and (4).

The boundary conditions to the system of equations are shown in Fig. 1. They are either essential boundary conditions when the velocity is specified, or natural boundary conditions when the traction is specified at the boundary.

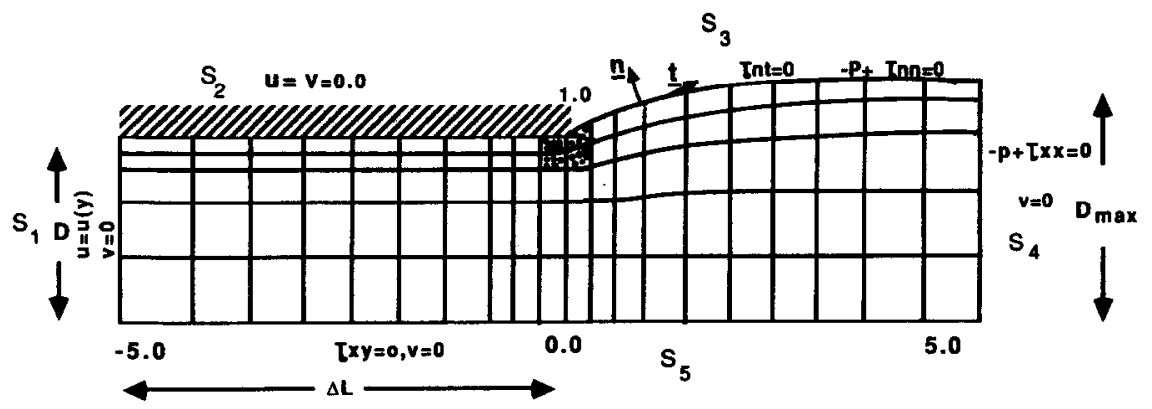

Fig. 1. The flow domain with the boundary conditions. 
Equations (2), (3), (4), and (7) are made dimensionless by choosing convenient units of stress $(\mu V / D)$, velocity $(V)$ and length $(D)$

$\nabla^{*} \cdot \boldsymbol{u}^{*}=0$

$\nabla^{*} \cdot\left(-p^{*} I+\tau_{1}^{*}+2 \frac{\mu_{2}}{\mu} D^{*}\right)=0$,

$\tau_{1}^{*}+\lambda_{1}^{*} \dot{\tau}_{1}^{*}-2\left(1-\frac{\mu_{2}}{\mu}\right) D^{*}=0$,

$n^{*} \cdot \boldsymbol{u}^{*}=0$.

The dimensionless number of interest here is the Weissenberg number

$W s=\lambda_{1} \gamma_{w}=\lambda_{1}^{*} \dot{\gamma}_{w}^{*}$,

where $\dot{\gamma}_{w}$ is the wall shear rate. The Deborah number for this flow is defined as $D e=W s / 3$. Equation (8c) reduces to the upper-convected Maxwell model when $\mu_{2}=0$ and to the Newtonian law when $\lambda_{1}=0$.

\section{Finite element formulation}

The velocity components $u$ and $v$ and the stress components $\tau_{x x}, \tau_{x y}$, and $\tau_{y y}$ are expanded in terms of biquadratic basis functions $\phi^{i}$, the pressure $p$ in terms of bilinear basis functions $\psi^{i}$, and the free surface elevation from the midplane in terms of quadratic basis functions $\hat{\phi}^{i}$ :

$$
\begin{aligned}
& u=\sum_{1}^{9} u_{i} \phi^{i}, v=\sum_{1}^{9} v_{i} \phi^{i}, \\
& \tau_{x x}=\sum_{1}^{9} \tau_{i} \phi^{i}, \tau_{x y}=\sum_{1}^{9} q_{i} \phi^{i}, \tau_{y y}=\sum_{1}^{9} r_{i} \phi^{i}, \\
& p=\sum_{1}^{4} p_{i} \psi^{i}, \\
& h=\sum_{1}^{3} h_{i} \hat{\phi}^{i} .
\end{aligned}
$$

Then eqns. (8a) to (8d) (with asterisk here and hereafter omitted) are weighted integrally by each of the basis functions, the divergence theorem is invoked and the resulted weighted residuals are required to vanish which determines the unknown coefficients $u_{i}, v_{i}, \tau_{i}, q_{i}, r_{i}, p_{i}$ and $h_{i}$ :

$R_{\mathrm{c}}^{i}=\int_{V}\left(\frac{\partial u}{\partial x}+\frac{\partial v}{\partial y}\right) \psi^{i} \mathrm{~d} V=0$ 


$$
\begin{aligned}
& R_{\mathrm{M}_{x}}^{i}=-\int_{V}\left|\left(-p+\tau_{1, x x}+2 \frac{\mu_{2}}{\mu} \frac{\partial u}{\partial x}\right) \frac{\partial \phi^{i}}{\partial x}+\left(\tau_{1, x y}+\frac{\mu_{2}}{\mu}\left(\frac{\partial u}{\partial y}+\frac{\partial v}{\partial x}\right)\right) \frac{\partial \phi^{i}}{\partial y}\right| \mathrm{d} V \\
& -\int_{s_{1}}\left(-p+\tau_{1, x x}+2 \frac{\mu_{2}}{\mu} \frac{\partial u}{\partial x}\right) \phi^{i} \mathrm{~d} s+\int_{s_{2}}\left(\tau_{1, x y}+\frac{\mu_{2}}{\mu}\left(\frac{\partial u}{\partial y}+\frac{\partial v}{\partial x}\right)\right) \phi^{i} \mathrm{~d} s \\
& +e_{x} \int_{s_{3}} n \cdot\left(-p I+\tau_{1}+\frac{\mu_{2}}{\mu} D\right) \phi^{i} \mathrm{~d} s+\int_{s_{4}}\left(-p+\tau_{1, x x}+2 \frac{\mu_{2}}{\mu} \frac{\partial u}{\partial x}\right) \phi^{i} \mathrm{~d} s \\
& -\int_{s_{5}}\left(\tau_{1, x y}+\frac{\mu_{2}}{\mu}\left(\frac{\partial u}{\partial y}+\frac{\partial v}{\partial x}\right)\right) \phi^{i} \mathrm{~d} s=0, \\
& R_{\mathrm{M}_{y}}^{i}=-\int_{V}\left|\left(-p+\tau_{1, y y}+2 \frac{\mu_{2}}{\mu} \frac{\partial v}{\partial y}\right) \frac{\partial \phi^{i}}{\partial y}+\left(\tau_{1, x y}+\frac{\mu_{2}}{\mu}\left(\frac{\partial u}{\partial y}+\frac{\partial v}{\partial y}\right)\right) \frac{\partial \phi^{i}}{\partial x}\right| \mathrm{d} V \\
& -\int_{s_{1}} \mid\left(\tau_{1, x y}+\frac{\mu_{2}}{\mu}\left(\frac{\partial u}{\partial y}+\frac{\partial v}{\partial x}\right) \mid \phi^{i} \mathrm{~d} s+\int_{s_{2}}\left(-p+\tau_{1, y y}+\frac{\mu_{2}}{\mu} \frac{\partial v}{\partial y}\right) \phi^{i} \mathrm{~d} s\right. \\
& +e_{y} \int_{s_{3}} \boldsymbol{n} \cdot\left(-p \boldsymbol{I}+\tau_{1}+\frac{\mu_{2}}{\mu} \boldsymbol{D}\right) \phi^{i} \mathrm{~d} s+\int_{s_{4}}\left(\tau_{1, x y}+\frac{\mu_{2}}{\mu}\left(\frac{\partial u}{\partial y}+\frac{\partial v}{\partial x}\right)\right) \phi^{i} \mathrm{~d} s \\
& -\int_{s_{s}}\left(-p+\tau_{1, y y}+\frac{\mu_{2}}{\mu} \frac{\partial v}{\partial y}\right) \phi^{i} \mathrm{~d} s=0, \\
& R_{\mathrm{C}_{1}}^{i}=\int_{V} \mid \tau_{1, x x}+\lambda_{1}\left(u \frac{\partial \tau_{1, x x}}{\partial x}+v \frac{\partial \tau_{1, x x}}{\partial y}\right)-\lambda_{1}\left(2 \tau_{1, x x} \frac{\partial u}{\partial x}+2 \tau_{1, x y} \frac{\partial u}{\partial y}\right) \\
& -2\left(1-\frac{\mu_{2}}{\mu}\right) \frac{\partial u}{\partial x} \mid \phi^{i} \mathrm{~d} V=0, \\
& R_{\mathrm{C}_{2}}^{i}=\int_{V} \int_{1, x y}+\lambda_{1}\left(u \frac{\partial \tau_{1, x y}}{\partial x}+v \frac{\partial \tau_{1, x y}}{\partial y}\right)-\lambda_{1}\left(\tau_{1, x x} \frac{\partial v}{\partial x}+\tau_{1, y y} \frac{\partial u}{\partial y}\right) \\
& -\left(1-\frac{\mu_{2}}{\mu}\right)\left(\frac{\partial u}{\partial y}+\frac{\partial v}{\partial x}\right) \mid \phi^{i} \mathrm{~d} V=0, \\
& R_{\mathrm{C}_{3}}^{i}=\int_{V} \tau_{1, y y}=\lambda_{1}\left(u \frac{\partial \tau_{1, y y}}{\partial x}+\frac{\partial \tau_{1, y y}}{\partial y}\right)-\lambda_{1}\left(2 \tau_{1, x y} \frac{\partial v}{\partial x}+2 \tau_{1, y y} \frac{\partial v}{\partial y}\right) \\
& -2\left(1-\frac{\mu_{2}}{\mu}\right) \frac{\partial v}{\partial y} \mid \phi^{i} \mathrm{~d} V=0, \\
& R_{\mathrm{k}}^{i}=\int_{s_{3}}\left(v-u \frac{\partial h}{\partial x}\right) \phi^{i} \mathrm{~d} s=0 .
\end{aligned}
$$


Equation (12) is replaced by the essential boundary condition $u=A(1-$ $\left.y^{2}\right)$ at the inlet $\left(S_{1}\right)$ and $u=0$ at the wall $\left(S_{2}\right)$ when the no-slip condition is applied. The third line integral is the tangential traction on the free surface $\left(S_{3}\right)$ and is zero when the surface tension is vanishingly small. The fourth boundary term is the normal traction at the outlet plane $\left(S_{4}\right)$ and is zero for the assumed plug flow. The fifth term is the tangential traction at the midplane of symmetry $\left(S_{5}\right)$ and is zero. Similar replacements or substitutions apply to the other momentum eqn. (13). No boundary conditions on the viscoelastic extra-stress at the inlet were found necessary. In fact, numerical experiments with stress inlet boundary conditions yielded wiggly stress profiles near the inlet. Nevertheless, solutions obtained with and without stress inlet conditions yielded identical predictions except the stress near the inlet. It appears that by not imposing essential boundary conditions on the stress at the inlet, allows the constitutive equation to hold within the flow domain and at the inlet where it serves as a mixed natural boundary condition. To apply the slip boundary condition the second boundary term of eqn. (12) is replaced by eqn. (1):

$\int_{s_{2}}\left(\tau_{1, x y}+\frac{\mu_{2}}{\mu}\left(\frac{\partial u}{\partial y}+\frac{\partial v}{\partial x}\right)\right) \phi^{i} \mathrm{~d} s=-\int_{s_{2}} \alpha u^{m} \phi^{i} \mathrm{~d} s$.

To account for surface tension the third boundary terms of both equations are substituted by the capillary traction [12]

$$
\begin{aligned}
\int_{s_{3}} n \cdot\left(-p I+\tau_{1}+\frac{\mu_{2}}{\mu} D\right) \phi^{i} \mathrm{~d} s & =-\int_{s_{3}} \frac{1}{C a} \phi^{i} \frac{\mathrm{d} t}{\mathrm{~d} s} \mathrm{~d} s \\
& =\frac{1}{C a} \int_{s_{3}} t \frac{\mathrm{d} \phi^{i}}{\mathrm{~d} s} \mathrm{~d} s-\frac{1}{C a}\left[\phi^{i} t_{1}-\phi^{i} t_{0}\right],
\end{aligned}
$$

where $n$ and $\boldsymbol{t}$ are the normal and tangent unit vectors to the free surface and $\mathrm{Ca}$ the capillary number defined as

$C a=\mu V / \sigma$,

where $\mu$ and $\sigma$ are the viscosity and the surface tersion of the viscoelastic solution respectively. In all the following calculation the surface tension was neglected unless otherwise stated.

To solve the system of the resulting nonlinear algebraic equations we used full Newton iteration

$\boldsymbol{S}^{(N+1)}=\boldsymbol{S}^{(N)}-\boldsymbol{J}\left(\boldsymbol{S}^{(N)}\right) \cdot \boldsymbol{R}\left(\boldsymbol{S}^{(N)}\right)$.

Here $S$ is the vector of the unknowns

$$
S=\left\{u_{1}, v_{1}, \tau_{1}, q_{1}, r_{1}, p_{1}, \ldots u_{n}, v_{n}, \tau_{n}, q_{n}, r_{n}, p_{n}, h_{n}\right\}
$$


and $J$ is the Jacobian of the residuals $R$ with respect to the nodal unknowns (coefficients in the expansions (10a) to (10d)). The simultaneous solution of eqns. (11) to (17) by Newton iteration provides rigorous distinction between convergence and divergence.

\section{Results}

\subsection{Newtonian liquid}

The results for Newtonian liquid were obtained with $\lambda_{1}=0$ in eqns. (11) to (17). The tessellation is shown in Fig. 1. The four square elements adjacent to the contact line, Figure 1, were subdivided further and further as follows. The predicted extrudate swell at these tessellations is plotted in Fig. 2. When no-slip was assumed the Newton iteration diverged before the solution became independent of the tessellation. When slip according to a linear relation shown in Fig. 2 was assumed, the Newton iteration converged at a finer tessellation near the singularity and the solution was independent of the tessellation. Thus the most appropriate boundary condition for a Newtonian liquid appears to be the one which accounts for some slip because it produces a solution which is independent of the tessellation. Our results are in agreement with the conclusions of Silliman and Scriven [9] arrived at by a different method.

\subsection{Upper-convected Maxwell liquid}

The results for this liquid were obtained with $\mu_{2} / \mu=0$ in eqns. (11) to (17). Figure 3 shows the maximum Weissenberg number at which the

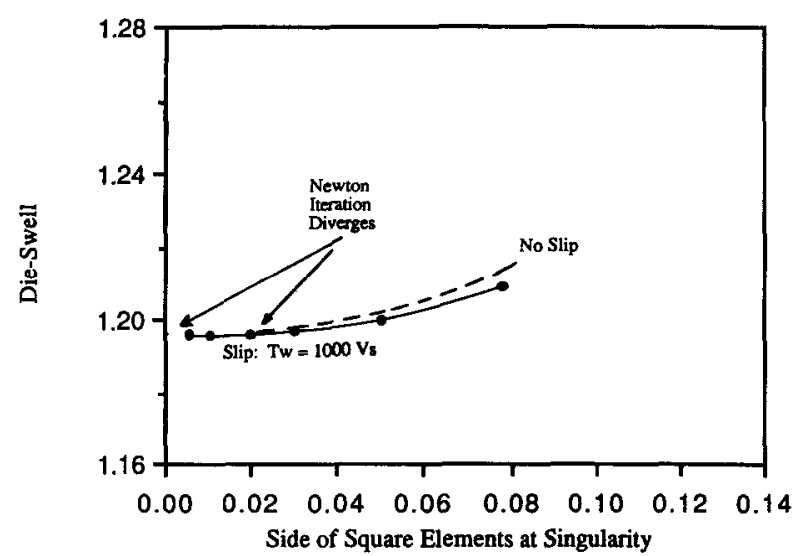

Fig. 2. Behavior of Newton iteration with mesh refinement with and without slip for Newtonian Liquid. 


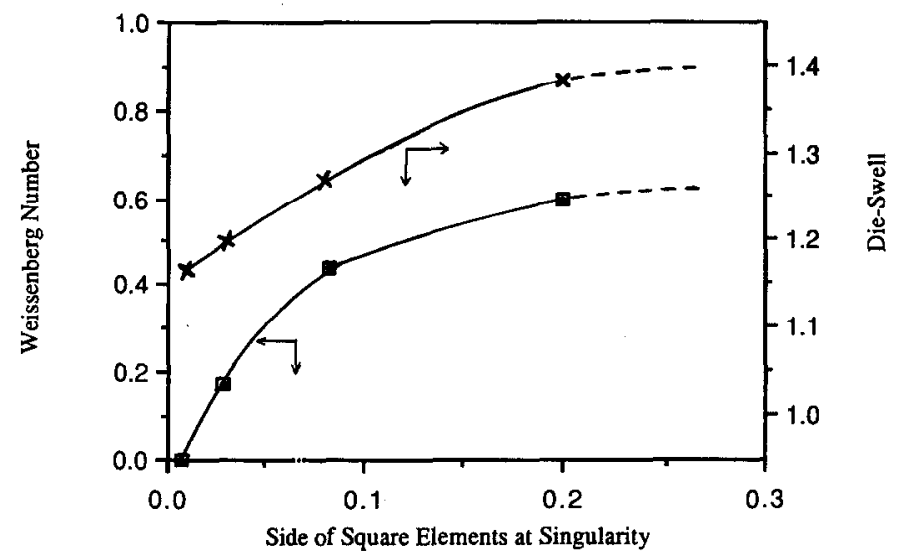

Fig. 3. Maximum Weissenberg number and die-swell predictions for upper-convected Maxwell liquid at several tessellations about the singularity with no-slip boundary condition.

Newton iteration converged quadratically with each of the tessellations used without slip. It is clear that the solution at any elasticity depended on the tessellation. Moreover, even for the Newtonian liquid of zero elasticity the Newton iteration diverged when the tessellation near the exit was refined further and further.

To proceed with the slip studies we used the tessellation of Fig. 1 which yields the correct value of 1.2 for the extrudate swell of Newtonian liquid. Figure 4 shows extrudate swell predictions obtained with five different slip laws:

(a) with practically no slip when, $\tau_{\mathrm{w}}=10^{6} V_{\mathrm{s}}$

(b) with a linear law, $\tau_{\mathrm{w}}=50 \mathrm{~V}_{\mathrm{s}}$,

which corresponds to Newtonian slip film

(c) with a superlinear law, $\tau_{\mathrm{w}}=400 V_{\mathrm{s}}^{2}$ or $\tau_{\mathrm{w}}=15000 \mathrm{~V}_{\mathrm{s}}^{4}$

which corresponds to shear thickening slip film.

(d) with a sublinear law, $\tau_{\mathrm{w}}=25 V_{\mathrm{s}}^{0.5}$

which corresponds to shear thinning slip film.

The equations for each of the layers are derived in Appendix A. Here $\tau_{\mathrm{w}}$ is the wall shear stress and $V_{\mathrm{s}}$ the velocity of the liquid at the solid wall, the slip velocity. The exponents were chosen arbitrarily. Then the coefficients were selected to predict correctly the Newtonian extrudate swell of value 1.200 [9]. The main conclusion from Fig. 4 is that the predictions of the upper-convected Maxwell model are extended from Weissenberg number 0.6 


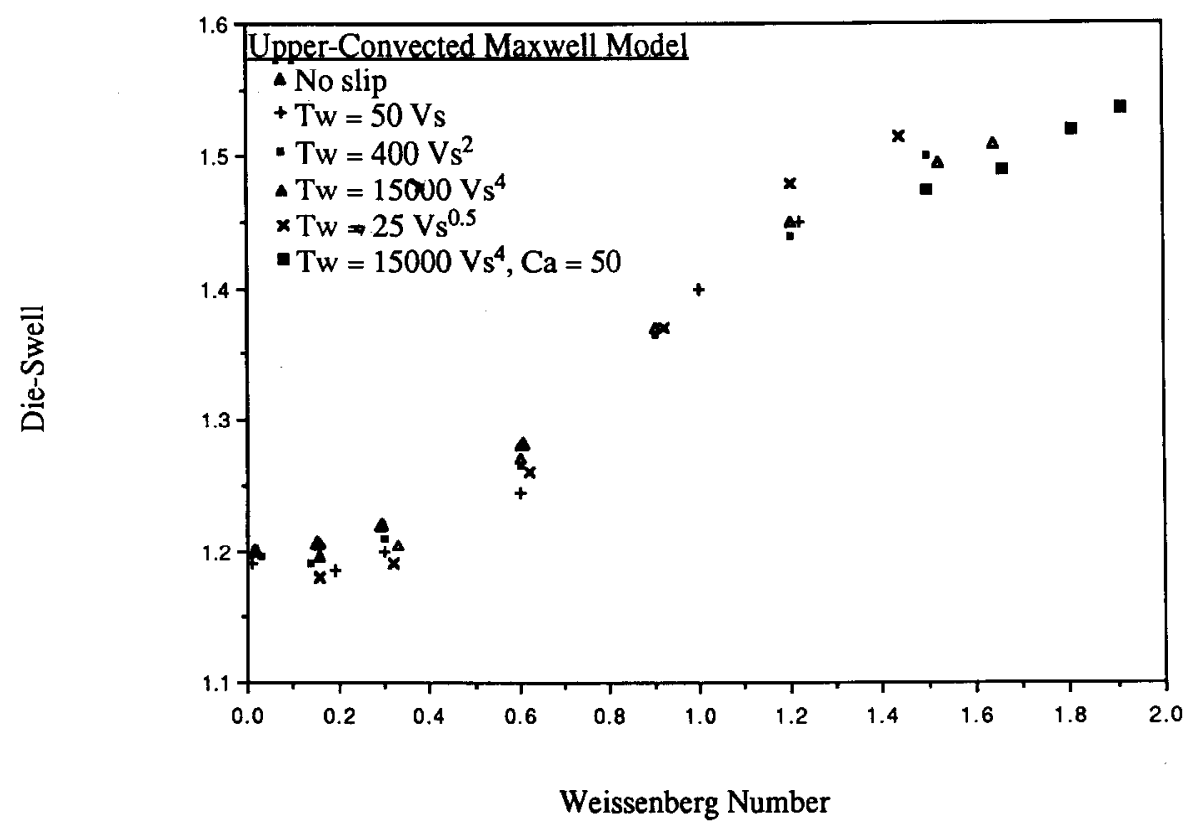

Fig. 4. Predicted die-swell for upper-convected Maxwell liquid with five different boundary conditions at the solid wall and with surface tension.

without slip to 1.7 with slip where the quadratic rate of convergence of the Newton iteration deteriorated and clearly diverged beyond Weissenberg 1.8 .

The White-Metzner extension of the upper convected Maxwell model with strain-thinning relaxation time and viscosity did not improve the calculations.

\subsection{Oldroyd-B liquid}

The results for this liquid were obtained with $\lambda_{1}=0$ and $\mu_{2} / \mu=0.1111$ in eqns. (11) to (17). Figure 5 shows the predicted extrudate swell with and without slip. Only slight improvements over the upper convected Maxwell model were obtained with either boundary condition.

\subsection{Surface tension}

The results for both viscoelastic liquids were slightly improved by accounting for surface tension by means of eqn. (18). The achieved improvement is shown in Figs. 4 and 5. The significance of the surface tension on 


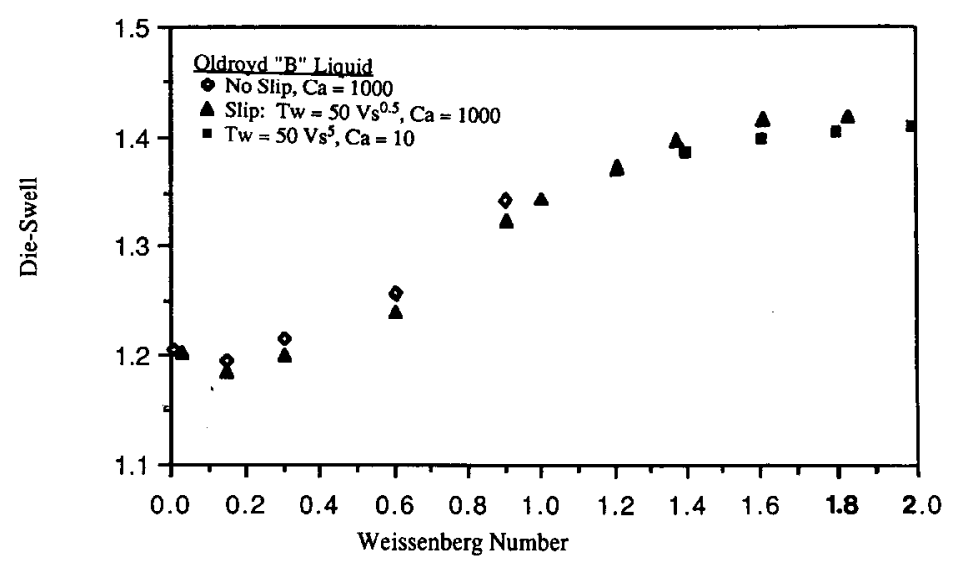

Fig. 5. Predicted die-swell for Oldroyd-B Liquid with and without slip and surface tension.

the extrudate swell is shown in Fig. 6 for the Oldroyd-B liquid. As expected surface tension surpresses the free surface and thus reduces extrudate swell.

\subsection{Retardation time or solvent viscosity}

Figure 7 shows the significance of the retardation time on the predicted extrudate swell and on the numerical stability. As expected by decreasing the viscoelastic to Newtonian character of the liquid, the extrudate swell decreases and the maximum stable Weissenberg number increases. The

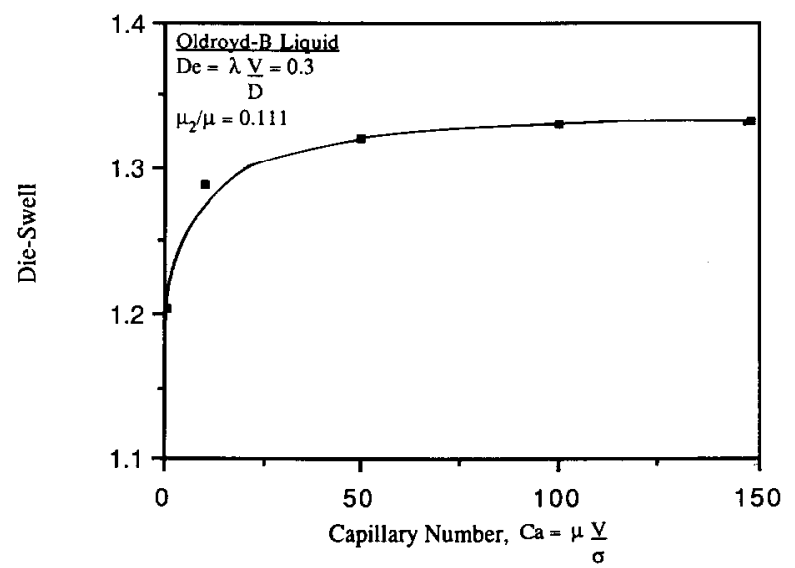

Fig. 6. Predicted die-swell for Oldroy-B liquid of $\lambda_{1} V / D=0.3$ at several Capillary numbers, $C a=\mu V / \sigma$. 


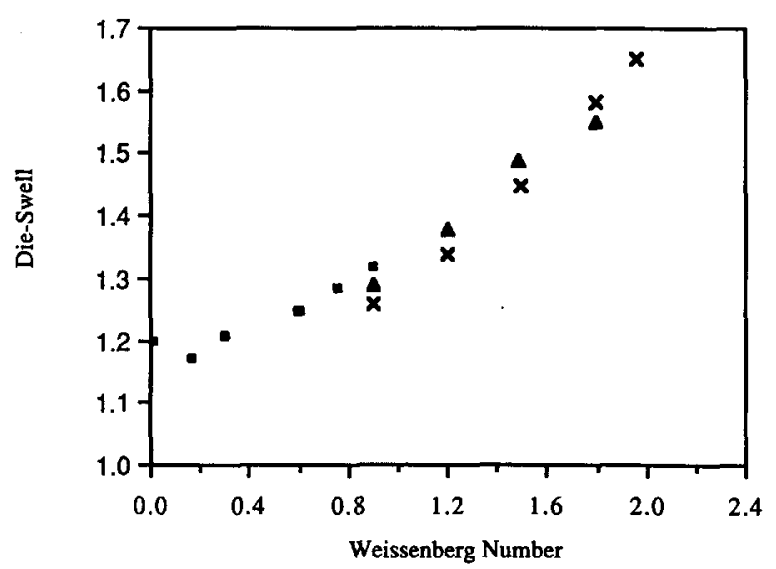

Fig. 7. Predicted die-swell and maximum Weissenberg number of an Oldroyd-B liquid of Newtonian to viscoelastic viscosity ratio $\mu_{1} / \mu_{2}$ of $1 / 8(\square), 2 / 7$ (A), and $4 / 5(X)$ with no-slip boundary conditions.

effects of the Newtonian viscosity on the predictions of the Oldroyd-B model at a fixed Weissenberg number are shown in Fig. 8.

\section{Discussion}

The conclusions drawn below apply to the particular method of solution we used by means of eqns. (11) to (17) and (21). This method is based on a global Newton iteration which calculates simultaneously velocities, pressure,

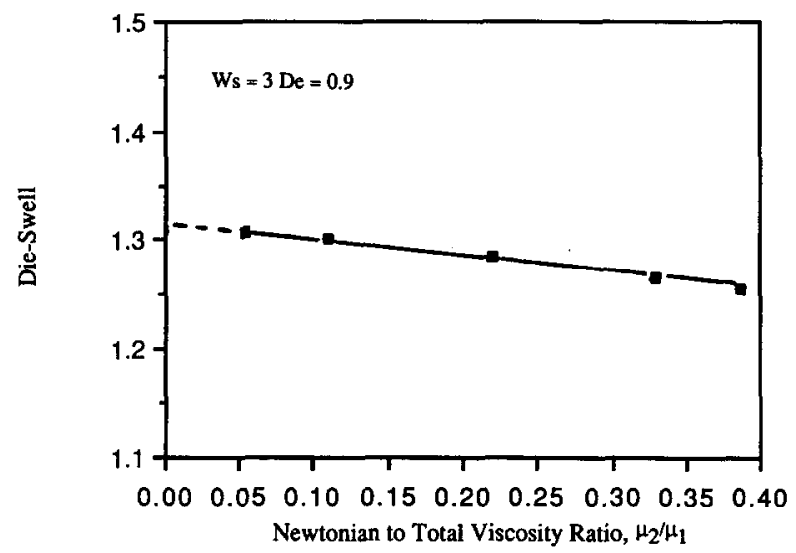

Fig. 8. Die-swell predictions of the Oldroyd-B model at constant Weissenberg number, $W s=3$. 


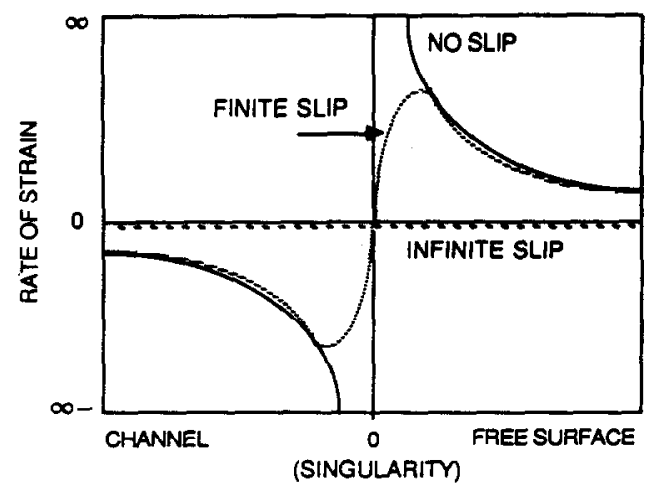

Fig. 9. Strain and stress along the solid wall and the free surface when there is no slip, finite slip and infinite slip.

stress, and free surface location. Thus the method is mathematically more rigorous than methods based on Newton under Picard iteration in the sense that it converges with quadratic rate and so it provides a clear distinction between convergence and divergence.

Figure 3 indicates that the strength of the singularity increases with the Weissenberg number. A theoretical explanation based on an analytical solution for a second order fluid at an infinitely large surface tension is given in Appendix B. Figures 2 and 4 suggest that the strength of the singularity decreases with the slip velocity. The analysis in Appendix $\mathrm{C}$ shows that in principle the singularity could be eliminated by slip but then the macroscopic predictions (e.g. pressure drop, extrudate swell, contact angle) would be altered significantly. Another limiting factor is the creation of maximum stresses, e.g. Fig. 9, in the vicinity of the singularity which is known to cause divergence. Thus the potential of the slip boundary condition as a means for alleviating the stress singularity is limited by pragmatic considerations.

The natural slip boundary condition, as expressed by eqn. (18), is the most appropriate because it produces numerical solutions which are independent of the tessellation and at relatively higher Weissenberg number. The divergence with mesh refinement for a viscoelastic liquid agrees with the results of Keunings [8] and Brown et al. [13] for contraction flow. Moreover, divergence with mesh refinement occurs even for Newtonian liquid.

The slip boundary condition improves calculations with the upper-convected Maxwell liquid by a factor of almost 3, from Weissenberg 0.6 without slip to 1.7 with slip. The same slip boundary condition improves calculations with the Oldroyd-B model by a factor of 2 , from Weissenberg 0.86 without slip to 1.866 with slip. It appears that the Oldroyd-B model embodies a 
mechanism equivalent to apparent slip, probably the retardation time (or the Newtonian viscosity $\mu_{2}$ of eqn. (6)), which reduces the stress at the singularity even without slip.

Another stabilizing factor is the surface tension even at Capillary number of values 100, which is typical of viscoelastic solutions, as Figs. 4 and 5 show. The stabilization is due to the fact that with surface tension included the viscoelastic stresses are not required to vanish just downstream yet.

A third stabilizing factor is the retardation time as shown by Fig. 7. By comparing eqns. (4) and (6) and given the relations (5c) and (5d) it is obvious that the retardation time $\lambda_{2}$ is proportional to the Newtonian viscosity $\mu_{2}$. Newtonian viscosity is known from standard linear elasticity theories to inhibit growth of instabilities by reducing the kinetic energy associated with them through viscous dissipation.

\subsection{Further justification of the slip boundary condition}

The stick-slip problem of Appendix B provides an excellent opportunity for further justification of the slip boundary contition. The stick-slip flow of the second order fluid was analysed by the finite element method of Section 3 with and without slip boundary conditions. The predicted velocity along the free surface is compared with the analytic solution, eqn. (B.2) of Appendix B, in Fig. 10. It is obvious that a slip boundary condition can

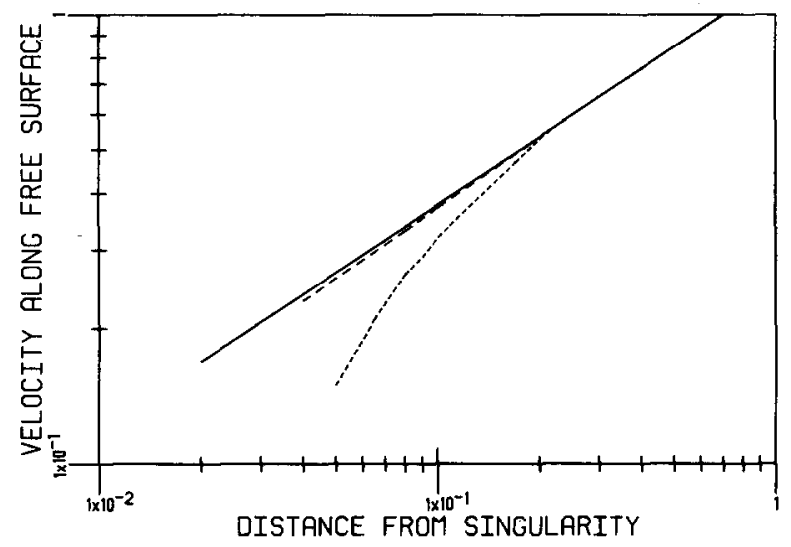

Fig. 10. Predicted velocity along the free surface in a stick-slip flow of a second order fluid (SOF), obtained with tessellation of Fig. 1, compared with the analytic solution: - . . . Analytic solution, eqn. (A.2) of Appendix A; --- Finite element predictions for SOF of $N_{1} V / \mu D=0.33$ and $N_{2}=0$ with a slip boundary condition, Equation (22b); $\cdots \cdots$ Finite Element Predictions for SOF of $N_{1} V / \mu D=0.33$ and $N_{2}=0$ with a no-slip boundary condition. 
yield the appropriate kinematics around the singularity. In contrast a no-slip boundary condition yields local kinematics which disagree with the analytic solution.

\subsection{Behavior of viscoelastic fluid particle at a singularity}

Both the finite element analysis and the asymptotic solutions substantiate Fig. 9 along the solid wall and the free surface of a singularity: when there is no slip the rate of strain and thus the stress is infinite (Appendix B). When there is slip a maximum stress is obtained (Appendix C). The Newton iteration diverges in both cases, however, at higher $W s$ for a maximum stress than for an infinite stress.

\section{Conclusions}

The use of a slip boundary condition in extrudate swell computations yields a tessellation-independent solution down to very fine mesh refinement near the singularity. The no-slip boundary condition yields a tessellation-dependent solution down to the point where the Newton iteration diverges. Further justification of the slip boundary condition is provided by its ability to reproduce the analytic solution to the stick-slip flow of a second order fluid in the vicinity of the singularity.

Slip improves computations with the upper convected Maxwell and the Oldroyd-B models. Inclusion of surface tension and Newtonian viscosity enhance some further stability. In all cases the stabilization is either due to the inhibition of the inhibition of stress growth by slip just upstream the contact line, or due to a free surface under tension, which produces nonvanishing viscoelastic stresses just downstream the contact line.

The main conclusion however is that, although slip velocity, viscosity and surface tension enhance some stability in numerical calculations, it is unlikely to advance viscoelastic computations significantly. In particular the behavior of viscoelastic constitutive equations in the vicinity of singularities where discontinuous deformation arises needs to be evaluated and then means to avoid the discontinuity or else to repair the constitutive equation need to be devised. A spectrum of relaxation times in both integral and differential constitutive equations is a good first step.

\section{Acknowledgement}

This work was supported by the Process and Reaction Engineering Program of the National Science Foundation, CBT-8504607. 


\section{Appendix A}

Justification of slip laws

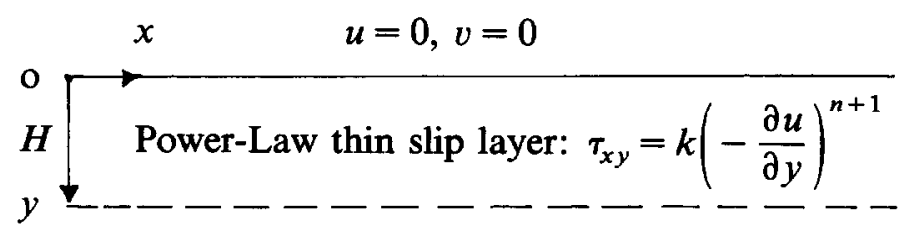

Bulk viscoelastic liquid: $\tau_{0}$

In thin layer: $C+\frac{\mathrm{d} P}{\mathrm{~d} x} y=\tau_{x y} \rightarrow C=\tau_{0}-\frac{\mathrm{d} P}{\mathrm{~d} y} H$,

$$
k\left(\frac{\partial u}{\partial y}\right)^{n+1}-\tau_{0}=\frac{\mathrm{d} P}{\mathrm{~d} x}(y-H) .
$$

Velocity in layer: $u=\left[\frac{\tau_{0}}{k}+\frac{1}{k} \frac{\mathrm{d} P}{\mathrm{~d} x}(y-H)\right]^{1+1 /(1+n)} /\left(1+\frac{1}{n+1}\right) \frac{\mathrm{d} P}{\mathrm{~d} x} \frac{1}{k}$

$$
-\left[\frac{\tau_{0}}{k}+\frac{1}{k} \frac{\mathrm{d} P}{\mathrm{~d} x} H\right]^{1+1 /(1+n)} /\left(1+\frac{1}{n+1}\right) \frac{1}{k} \frac{\mathrm{d} P}{\mathrm{~d} x} .
$$

Slip velocity: $V=u(y=H)=\left(\frac{\tau_{0}}{k}\right)^{1+1 /(n+1)}-\left(\frac{\tau_{0}}{k}+\frac{1}{k} \frac{\mathrm{d} P}{\mathrm{~d} x} H\right)^{1+1 /(n+1)}$

$$
\begin{gathered}
/\left(1+\frac{1}{n+1}\right) \frac{\mathrm{d} P}{\mathrm{~d} x} \frac{1}{k}, \\
V=\frac{\frac{(n+2)}{(n+1)}\left(\frac{\tau_{0}}{k}\right)^{1 /(n+1)} \frac{1}{k} \frac{\mathrm{d} P}{\mathrm{~d} x} H}{\left(\frac{n+2}{n+1}\right) \frac{\mathrm{d} P}{\mathrm{~d} x} \frac{1}{k}}=H\left(\frac{\tau_{0}}{k}\right)^{1 /(n+1)} .
\end{gathered}
$$

Slip law: $\tau_{0}=k\left(\frac{V}{H}\right)^{n+1}\left\{\begin{array}{l}n<0, \text { Thinning, } \\ n=0, \text { Newtonian, } \\ n>0, \text { Thickening. }\end{array}\right.$

The case $n=0$ was derived earlier by Joseph [14]. 


\section{Appendix B}

Solution to stick-slip flow [15] for second order fluid (SOF).

\section{Singularity}

Solid Wall: $U_{r}=U_{\theta}=0$

Free Surface: $U_{\theta}=\tau_{r \theta}=0$

Flow

$\rightarrow$

$\theta$

The kinematics around the singularity with respect to a polar system $(r, \theta)$ with its origin at the singularity are after Moffat [16]:

$\psi=r^{3 / 2} \mathrm{f}(\alpha), f(\theta)=A \sin \theta \sin \frac{\theta}{2}$,

$U_{r}=\frac{1}{r} \frac{\partial \psi}{\partial \theta}=r^{1 / 2} f^{\prime}(\theta)$,

$U_{\theta}=-\frac{\partial \psi}{\partial r}=-\frac{3}{2} r^{1 / 2} f(\theta)$.

The Giesekus-Tanner theorem [17] applies here if no slip along the solid wall is assumed $\left(\psi_{y y}=\right.$ zero along the free surface guarantees shear stress $\tau_{r \theta}=0$ for both Newtonian and SOF liquids). Thus close to the singularity the stresses for the SOF are

$\tau_{r r}=\mu r^{-1 / 2} f^{\prime}+N_{1} r^{-1} F_{1}\left(f, f^{\prime}, f^{\prime \prime}\right)$,

$\tau_{\theta \theta}=-\mu r^{-1 / 2} f^{\prime}+N_{1} r^{-1} F_{2}\left(f, f^{\prime}, f^{\prime \prime}, f^{\prime \prime \prime}\right)$,

$\tau_{r \theta}=\tau_{\theta r}=\frac{3}{4} \mu r^{-1 / 2}\left(f+\frac{4}{3} f^{\prime}\right)+N_{1} r^{-1} F_{3}\left(f, f^{\prime}, f^{\prime \prime}, f^{\prime \prime \prime}\right)$,

where $\mu$ is the constant Newtonian viscosity, $N_{1}$ the constant first normal stress coefficient (the second normal stress coefficient $\mathrm{N}_{2}$ assumed zero without loss of generality), and $F_{i}$ known functions of $f(\theta)$ and its derivatives. It is clear that for Newtonian liquid $\left(N_{1}=0\right)$

$\tau_{i j} \alpha r^{-1 / 2}$,

and for a second order fluid $\left(N_{1} \neq 0, N_{2}=0\right)$

$\tau_{i j} \alpha r^{-1}$.

Thus elasticity incorporated in $N_{1}=0$ increases the strength of the singularity from $r^{-1 / 2}$ to $r^{-1}$.

A similar analysis for a SOF was carried out independently by Lipscomb et al. [18]. 


\section{Appendix C}

Solution to stick-slip flow with slip-boundary condition

\begin{tabular}{ccccc} 
Solid Wall & & \\
|||||||||| & $v=0$ & |||||| $\mid$ & $v=0$ & Free Surface \\
\hline$\tau_{r \theta}=\alpha \mathbf{u}^{\beta}$ & 0 & $\tau_{r \theta}=0$ &
\end{tabular}

Assume a solution $\psi=r^{\lambda} \mathrm{f}_{\lambda}(\theta)$.

Then $u=r^{\lambda-1} \mathrm{f}_{\lambda}^{1}(\alpha)$ and $v=\lambda r^{\lambda-1} f_{\lambda}(\theta)$,

where $\mathrm{f}_{\lambda}(\theta)=A \cos \lambda \theta+B \sin \lambda \theta+C \cos (\lambda-2) \theta+D \sin (\lambda-2) \theta$.

At $\theta=0, v=0, A=-C$;

At $\theta=\pi, v=0$;

At $\theta=\pi, \tau_{\mathrm{r} \theta}=0$;

under these conditions

$$
\begin{aligned}
v= & \left\{(\cos \lambda \theta-\cos (\lambda-2) \theta)+\frac{\cos (\lambda-2) \pi}{\sin (\lambda-2) \pi} \sin (\lambda-2) \theta\right. \\
& \left.-\frac{\cos \lambda \pi}{\sin \lambda \pi} \sin \lambda \theta\right\}\left(-\lambda r^{\lambda-1}\right), \\
u= & \left\{(-\lambda \sin \lambda \theta+(\lambda-2) \sin (\lambda-2) \theta)+\frac{\cos (\lambda-2) \pi}{\sin (\lambda-1) \pi}(\lambda-2) \cos (\lambda-2) \theta\right. \\
& \left.-\frac{\cos \lambda \pi}{\sin \lambda \pi} \lambda \cos \lambda \theta\right\}\left(\mathrm{r}^{\lambda-1}\right), \\
\tau_{r \theta}= & \left\{-\lambda^{2} \cos \lambda \theta+(\lambda-2)^{2} \cos (\lambda-2) \theta+\frac{\cos (\lambda-2) \pi}{\sin (\lambda-2)}(\lambda-2)^{2} \sin (\lambda-2) \theta\right. \\
& \left.+\frac{\cos \lambda \pi}{\sin \lambda \pi} \lambda^{2} \sin \lambda \theta\right\}\left(\mathrm{r}^{\lambda-2}\right) .
\end{aligned}
$$

At $\theta=0$

$u=r^{\lambda-1} A\left\{0+\frac{\cos (\lambda-2) \pi}{\sin (\lambda-2) \pi}(\lambda-2)-\frac{\cos \lambda \pi}{\sin \lambda} \lambda\right\}$

and

$\tau_{r \theta}=A r^{\lambda-2}\left\{-\lambda^{2}+\lambda^{2}-4 \lambda+4\right\}$. 
and the slip law yields

$\tau_{r \theta}=\alpha u^{\beta}$ with $\beta=(\lambda-2) /(\lambda-1)$.

Substitution of $u$ and $\tau_{r \theta}$ in (C.3) and (C.4) yields

$\{-4 \lambda+4\}=\alpha\left\{-2 \frac{\cos \lambda \pi}{\sin \lambda \pi}\right\}^{(\lambda-2) /(\lambda-1)}$,

where $\alpha$ is an arbitrary constant

$\{-4 \lambda+4\}=\alpha\left\{-2 \frac{\cos \lambda \pi}{\sin \lambda \pi}\right\}^{(\lambda-2) /(\lambda-1)}$.

Equation (B.7) has many solutions with $\lambda>2$, for which the stress $\tau_{r \theta}$ is not singular, dependent on the value of the slip coefficient, $\alpha$. Thus slip boundary conditions of the form of (B.5) alleviate the singularity in Newtonian and second order liquids and in this slip layers of other liquids induced near solid boundaries. However, the function $\tau_{r \theta}$ vs. $r$ is forced to go through a maximum with slip and the Newton iteration still diverges. Figure 9 , which is obtained by several investigators shows exactly this divergence. The Newton iteration does not only diverge when infinite stresses are encountered but also when maximum stresses are created by slip. In general the Newton iteration diverges at maximum stresses or deformations extremes of which are infinite stresses and deformations.

\section{References}

1 M.J. Crochet and K. Walters, Ann. Rev. Fluid Mech., 15 (1983).

2 M.J. Crochet and R. Keunings, J. Non-Newtonian Fluid Mech., 10 (1982) 339.

3 X.L. Luo and R.I. Tanner, J. Non-Newtonian Fluid Mech., 21 (1986) 179.

4 B. Caswell and K. Viriyayuthakorn, J. Non-Newtonian Fluid Mech., 12 (1983) 12.

5 B. Bernstein, D.S. Malkus and E.T. Olsen, Int. J. Num. Meth. Fluids, 5 (1985) 45.

6 T.C. Papanastasiou, L.E. Scriven and C.W. Macosko, J. Non-Newtonian Fluid Mech., 22 (1987) 271.

7 P.W. Yeh, M.E. Kim-E., R.C. Armstrong and R.A. Brown, J. Non-Newtonian Fluid Mech., 16 (1984) 173.

8 R. Keunings, J. Non-Newtonian Fluid Mech., 20 (1986) 209

9 W.T. Silliman and L.E. Scriven, J. Comp. Phys., 34 (1980) 287.

10 A.M. Kraynik and W.R. Schowalter, J. Rheol., 25 (1981) 97.

11 Y. Cohen and A.B. Metzner, J. Rheol., 29 (1985) 67.

12 K.J. Ruschak, Int. J. Num. Meth. Eng., 15 (1980) 639.

13 R.A. Brown, R.C. Armstrong, A.N. Beris, and P.-W. Yeh, Comp. Meth. Appl. Mech. Eng., 58 (1986) 201.

14 D.D. Joseph, J. Phys. Fluids, 23 (1980) 2356.

15 S. Richardson, Proc. Comb. Phil. Soc., 67 (1970) 477.

16 H.K. Moffat, Fluid Mech., 18 (1963) 1.

17 H. Giesekus, Rheol. Acta, 3 (1963) 59; R.I. Tanner, Phys. Fluids, 9 (1966) 1246.

18 G.G. Lipscomb, R. Keunings and M.M. Denn, J. Non-Newtonian Fluid Mech., 24 (1987) 85. 